\title{
Multi-mirror telescope confounds the sceptics
}

David Dickson reports on a telescope with a radical new design which is to be officially inaugurated in New Mexico next week

From the main road it looks like a huge, giftwrapped Christmas present, perched precariously on the summit of the 8,500-ft Mount Hopkins, 25 miles south of Tucson, Arizona. Only as you cover the last, steep stretch of the approach road and begin to glimpse the apparatus behind the rolledback doors, does the building's

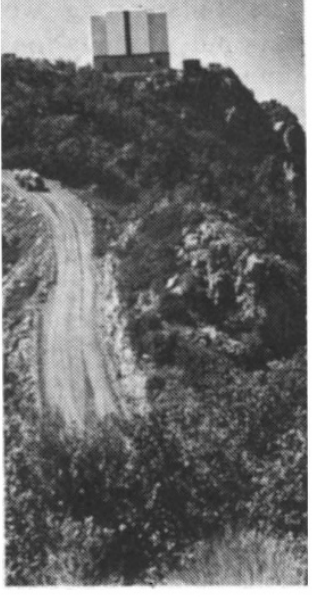

function as an observatory become apparent-despite the absence of the conventional split dome.

Even then, the surprises are not over. Rather than the long, carefully balanced cylindrical telescope that one expects, there is a squat, almost cubeshaped construction with six identical mirrors hidden in a complex of steel girders, the whole apparatus supported by a massive steel yoke.

The Multi-Mirror Telescope, has been built jointly by the Smithsonian Institution and the University of Arizona, and is to be officially inaugurated next week. Attending the dedication ceremony may well be some of those who were initially sceptical about the feasibility of combining six separate reflecting mirrors in a single telescope, a step which the designers claim to be the first major departure from conventional optical telescope construction in more than a century.

"It is a radical design, and it met with an enormous amount of scepticism in the beginning. Even those involved in the project found they had to overcome some of their own biases and prejudices", says Professor William F. Hoffmann, of the University of Arizona's department of astronomy. "Many doubted whether it was practical to trade off the cost of a single, large primary mirror against the complexity of the control and alignment systems needed to operate a number of smaller mirrors simultaneously. And even if the trade-off proved to be worth it, there were doubts about whether the control system could be made to work adequately-and to run routinely."

Some of these reservations-and in particular the last one-have yet to be answered. But preliminary viewings have already been successful, even though the telescope has to be operated manually until the computerised control system is linked up. Professor Hoffmann says he does not foresee any major problems.

There are three radical innovations in the MMT design, each of which is being closely followed elsewhere as suggestion new directions in telescope construction. The MMT is really a combination of six parallel Cassagrain telescopes, each with its own 1.8-metre primary mirror and its own secondary mirror, with the separate images focused to a single point. This results in a light collecting capability equivalent to a 4.5-metre telescope-still behind the 5-metre telescope on Mount Palomar and the Russian 6-metre telescope, but its compact design means that, at $\$ 7.5$ million (in 1975 prices), the MMT cost less than a third of a conventional design.

Laser beams align the mirrors, using the MMT's guide-alignment telescope as a collimator for a laser-generated point source. The beam is split into six components, each used to keep a check on one of the mirrors and control its fine-adjustment mechanisms. "The problem is comparable to getting six headstrong ballerinas to dance as if they were one," says one of the project directors.

MMT may well serve as a prototype for a new generation of optical telescopes, avoiding the increasingly prohibitive costs of large primary mirrors. Already a multi-mirror design is being considered as one option for a 10-metre telescope being planned by the University of California. Scientists at the neighbouring Kitt Peak National Observatory are exploring a similar approach to a 25-metre instrument.

The second design innovation is the

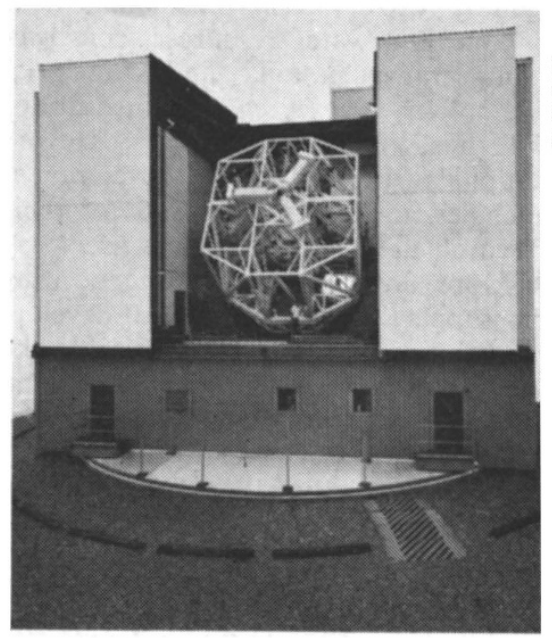

Complex of steel girders hides the mirrors mounting. Most conventional telescopes use an equatorial mount, meaning that movement about only one axis is required to compensate for the rotation of the Earth. The MMT, in contrast, uses an alt-azimuth mount, the horizontal axis being provided by a large steel yoke which itself turns on a vertical axis. Keeping track of an object during viewing means rotating the telescope about both axes simultaneously, a process which is controlled by a special mini-computer.

Both the compact design of the telescope, and the alt-azimuth mounting, led to the concept of a rectangular housing. "Any dome is, in its own geometry, azimuthal, but with an equatorially-mounted telescope you need the extra swing-space within the dome," says Professor Hoffmann. "With the alt-azimuth mount, motion is either up and down or circular, so saving space inside the dome is no longer necessary. A rectangular space then became much more convenient than a spherical one."

Professor Hoffmann says that although when first suggested the fork mounting and the rectangular building were considerable innovations, it turned out to be so practical that he thinks "people will soon find that the hemispherical dome and the equatorial mount are no longer attractive". Scientists from the Australian National University are already contemplating using this approach for a new telescope on Mount Stromlo, and others are expected to follow.

When it becomes fully operational, the MMT, which will be able to operate in both visible and infra-red parts of the spectrum, will have a number of uses. It will be able to carry out infrared photometry that could help scientists identify materials on the surface of Pluto and the satellites of Uranus and Neptune, and also provide detailed measurements of highly red-shifted optical spectral lines from distant quasistellar objects.

Another major application will be in spectroscopy. By projecting the images from the six main mirrors along a line, rather than on to a single spot, the MMT will be able to compensate for the light lost when the single image from a conventional telescope is focused on a single slit.

With other ground-based facilitiespanticularly those at Kitt Peak-increasingly in demand, there will be no shortage of users for the MMT. "They are already beating a path to our door," says Professor Hoffmann. Initial users will be those from the Smithsonian and the University of Arizona. Subsequently, applications for experiments will be open to all comers. 